\title{
A Pooled Analysis of Coronary Arterial Patency and Left Ventricular Function After Intravenous Thrombolysis for Acute Myocardial Infarction
}

\author{
Christopher B. Granger, MD, Harvey D. White, MD, Eric R. Bates, MD, \\ E. Magnus Ohman, MD, and Robert M. Califf, MD
}

\begin{abstract}
Individual studies of patency rates and left ventricular (LV) function after thrombolysis have generally been limited by small numbers of observations, wide confidence intervals, and limIted numbers of time points. To obtain a more reliable estimate of pattems of patency and LV ejection fraction, a systematic overview of angiographic studies was performed after intravenous thrombolytic thorapy. A total of 14,124 anglographic observations from 58 studies evaluating patency after no thrombolytic agent, streptokinase, standard dose tissue-type plasminogen activator (tPA), accelerated dose t-PA, or anistreplase (anisoylated plasminogen streptokinase activator complex [APSAC]) were included. At 60 and 90 minutes, streptokinase had the lowest patency rates of $48 \%$ and $51 \%$, respectively, standard dose tPA and APSAC had similar Intermediate rates of approximately $60 \%$ and $70 \%$, and accelerated tPA had the highest patency rates of $74 \%$ and $84 \%$. By 2 to 3 hours and
\end{abstract} longer, the patency rates were similar for the varlous regimens. Reocclusion rates in studies including 1,172 patients randomized to t-PA versus t? nomfibrin-specific agent were higher after t-PA (13.4\% vs 8.0\%, p = 0.002). Ten studies onrolling 4,088 patients treated with thrombolytic therapy versus control demonstrated a modest improvement in mean LV ejection fraction in the thrombolytic group at each of the times after thrombolytic therapy: hour 4, day 1, day 4, day 7 to 10, and day 10 to 28 after thrombolysis. By 4 days, mean ejection fraction was $53 \%$ versus 47\% (thrombolytic vs control therapy, $p<0.01$ ); by 10 to 28 days it was $54.1 \%$ and $51.5 \%$, respectively. In conclusion, this pooled analysis shows that accelerated t-PA resulted in higher 90 -minute coronary arterial patency rates than other standard regimens, but that by 2 to 3 hours the rates were similar, and that reocclusion rates were higher after t-PA than nonfibrin-

From the Division of Cardiology, Department of Medicine, Duke University Medical Center, Durham, North Carolina; Cardiovascular Research and Coronary Care Units, Green Lane Hospital, Auckland, New Zealand; and Division of Cardiology, Department of Medicine, University of Michigan, Ann Arbor, Michigan. This study was supported in part by Research Grant HL-36587 from the National Heart, Lung, and Blood Institute, Bethesda, Maryland, and Research Grant HS-05636 from the Agency for Health Care Policy and Research, Rockville, Maryland. Manuscript received December 8, 1993; revised manuscript received August 1, 1994, and accepted August 3.

Address for reprints: Christopher B. Granger, MD, Box 3409, Duke University Medical Center, Durham, North Carolina 27710. specific agents. Thrombolytic therapy resulted in only a small improvement in global LV function compared with results in the control group, which was fully apparent by 4 days after treatment.

(Am J Cardiol 1994;74:1220-1228)

$\mathrm{R}$ eperfusion, sustained early patency, prevention of reocclusion, and preservation of left ventricular (LV) function are thought to be the basis for the beneficial effect of thrombolytic therapy in patients with acute myocardial infarction. Although many angiographic studies have assessed infarct artery patency and LV function in patients receiving thrombolytic therapy, the results of individual studies have generally been limited by wide confidence intervals because each study involved small numbers of patients. Moreover, individual angiographic studies have usually collected data at only 1 or 2 time points, failing to provide sequential information about infarct artery patency or LV function over time. This study provides a more reliable estimate of patterns of perfusion, reocclusion, and LV ejection fraction after frequently used intravenous thrombolytic regimens by pooling angiographic data from all available studies.

\section{METHODS}

Studies evaluating intravenous thrombolytic therapy with acute or follow-up angiography were identified by computer-aided search (Medline), careful review of reference lists of angiographic study reports, and related review articles, review of abstracts from the American Heart Association and American College of Cardiology meetings, and discussions with colleagues involved in this type of investigation. When different publications were found reporting data on the same group of patients, the more complete report was selected.

Studies that fulfilled the following criteria were included: $\geq 20$ patients were enrolled, prospective design, and reporting of the total number of patients initially treated as well as the number who underwent angiography or ventriculography, or both. Publication of the studies that were included spanned the years 1984 to 1993 . For the patency analysis, studies were included if they used intravenous streptokinase, standard dose tissuetype plasminogen activator (t-PA), accelerated dose tPA, anistreplase (anisoylated plasminogen streptokinase activator complex [APSAC]), or no thrombolytic agent, and if they reported the number of patients who had patent infarct-related arteries and the mean time of angiography after initiation of treatment. Studies that used the following doses for each agent were included: streptokinase, $>1.0$ million $\mathrm{U}$; standard t-PA, 0.75 to 1.5 
$\mathrm{mg} / \mathrm{kg}$ or 70 to $100 \mathrm{mg}$ single chain and not meeting criteria for accelerated dosing; accelerated t-PA, $>70 \mathrm{mg}$ over 1 hour or $100 \mathrm{mg}$ over 90 minutes, or both; or APSAC, $30 \mathrm{U}$ over 2 to 5 minutes. For the analysis of reocclusion, studies were included that randomized patients to intravenous t-PA versus either streptokinase, APSAC, or urokinase, and reported early (approximately 90 minutes after initiation of thrombolytic therapy) and follow-up ( $\geq 24$ hours after initiation of thrombolytic therapy) infarct-related artery patency. The largest single angiographic study after thrombolytic therapy, the Global Utilization of Streptokinase and t-PA for Occluded Arteries (GUSTO) angiographic substudy, ${ }^{1}$ had adequate power on its own to reliably determine patency rates and evaluate patency at serial time points after thrombolytic therapy, and thus was not included in this pooled analysis.

For LV ejection fraction analysis, studies were included that randomized patients to intravenous thrombolytic therapy versus control therapy, and reported mean time to ventriculography, mean LV ejection fraction, standard deviation of ejection fraction, and number of patients included in the determination of mean ejection fraction.
Infarct-related artery patency analysis: Mean angiographic time points were pooled into 5 periods after the initiation of thrombolytic therapy: 1 hour (45 to 74 minutes), 90 minutes ( 75 to 104 minutes), 2 to 3 hours (105 to 195 minutes), 1 day (18 to 48 hours), and 3 to 21 days. For studies in which patients were not treated with thrombolytic therapy, the time interval was measured from initiation of placebo treatment or heparin. These time groupings were selected because the studies used angiographic end points clustered around them.

Reocclusion analysis: Reocclusion rate was calculated as the number of patients with an angiographically occluded infarct-related artery who had had a patent infarct-related artery at the end of a prior " 90 -minute" catheterization, divided by the total number of patients who had a patent artery at the 90 -minute catheterization. The pooled reocclusion rate was calculated as the sum of the patients with reocclusion divided by the sum of the patients with originally patent infarct-related arteries.

Left ventricular ejectlon fraction analysis: For LV ejection fraction analysis, ventriculographic time points were pooled into 5 time periods: baseline ( 0 hours), 4 hours, 4 days, 7 to 10 days, and 10 to 28 days, at which time there was a clustering of ventriculographic deter-

\begin{tabular}{|c|c|c|c|c|c|}
\hline Study & $\begin{array}{l}\text { Number } \\
\text { of Patients }\end{array}$ & $\begin{array}{l}\text { Time to } \\
\text { Treatment } \\
\text { (min) }\end{array}$ & $\begin{array}{l}\text { Mean } \\
\text { Cath. } \\
\text { Time }\end{array}$ & Patency & $95 \% \mathrm{Cl}$ \\
\hline $\begin{array}{l}\text { Baseline } \\
\text { Anderson (1984) } \\
\text { TIMI-I (1987) } \\
\text { Timmis (1987) }\end{array}$ & $\begin{array}{r}23 \\
289 \\
40\end{array}$ & $\begin{array}{l}168 \\
285 \\
186\end{array}$ & $\begin{array}{l}0 \min \\
0 \min \\
0 \min \end{array}$ & $\begin{array}{c}9 \%(2 / 23) \\
20 \%(57 / 289) \\
28 \%(11 / 40)\end{array}$ & \\
\hline Pooled & 352 & & & $20 \%(70 / 352)$ & $16 \%-24 \%$ \\
\hline $\begin{array}{l}1 \text { hour } \\
\text { Collen (1984) } \\
\text { Topol (1987) } \\
\text { Cribier (1986) }\end{array}$ & $\begin{array}{l}14 \\
25 \\
23\end{array}$ & $\begin{array}{l}284 \\
228 \\
208\end{array}$ & $\begin{array}{l}45 \min \\
68 \min \\
74 \mathrm{~min}\end{array}$ & $\begin{array}{r}7 \%(1 / 14) \\
13 \%(3 / 23) \\
22 \%(5 / 23)\end{array}$ & \\
\hline Pooled & 62 & & & $15 \%(9 / 60)$ & $6 \%-24 \%$ \\
\hline $\begin{array}{l}90 \text { minute } \\
\text { ECSG-1 (1985) }\end{array}$ & 65 & 198 & $75-90 \mathrm{~min}$ & $21 \%(13 / 62)$ & $11 \%-31 \%$ \\
\hline $\begin{array}{l}2-3 \text { hour } \\
\text { Guerci (1987) }\end{array}$ & 66 & 192 & 2.5 hours & $24 \%(15 / 62)$ & $14 \%-35 \%$ \\
\hline $\begin{array}{l}1 \text { day } \\
\text { TPAT (1989) } \\
\text { Durand (1987) }\end{array}$ & $\begin{array}{l}56 \\
29\end{array}$ & $\begin{array}{l}180 \\
149\end{array}$ & $\begin{array}{l}17 \text { hours } \\
35 \text { hours }\end{array}$ & $\begin{array}{l}29 \%(7 / 24) \\
13 \%(3 / 24)\end{array}$ & \\
\hline Pooled & 85 & & & $21 \%(10 / 48)$ & $9 \%-32 \%$ \\
\hline $\begin{array}{l}\text { 3-21 day } \\
\text { Bassand (1989) } \\
\text { NHFA (1988) } \\
\text { TPAT (1989) } \\
\text { Kennedy (1988) } \\
\text { ECSG-4 (1988) } \\
\text { White (1987) } \\
\text { O'Rourke (1988) } \\
\text { Bassand (1987) }\end{array}$ & $\begin{array}{r}119 \\
71 \\
56 \\
177 \\
366 \\
112 \\
71 \\
55\end{array}$ & $\begin{array}{l}188 \\
195 \\
180 \\
210 \\
168 \\
180 \\
114 \\
190\end{array}$ & $\begin{array}{c}4.1 \text { days } \\
5-7 \text { days } \\
10 \text { days } \\
10 \text { days } \\
10-21 \text { days } \\
21 \text { days } \\
21 \text { days } \\
21 \text { days }\end{array}$ & $\begin{array}{l}36 \%(38 / 105) \\
41 \%(26 / 64) \\
59 \%(17 / 29) \\
45 \%(47 / 105) \\
78 \%(259 / 334) \\
54 \%(50 / 92) \\
64 \%(40 / 63) \\
67 \%(31 / 46)\end{array}$ & \\
\hline Pooled & 1,027 & & & $61 \%(508 / 838)$ & $57 \%-64 \%$ \\
\hline $\begin{array}{l}\mathrm{Cl}=\text { confldence inte } \\
\text { Activator in Acute Myc } \\
\text { angiography; NHFA = } \\
\text { until initiation of throm } \\
\text { Plasminogen Activator }\end{array}$ & $\begin{array}{l}\text { ECSG = Eur } \\
\text { I Infarction t } \\
\text { nal Heart Fo } \\
\text { or control tr } \\
\text { nto trial. }\end{array}$ & $\begin{array}{l}\text { Cooperative } \\
\text { lean Cath. T } \\
\text { on of Austra } \\
\text { ent; TIMI = T }\end{array}$ & $\begin{array}{l}\text { dy Group for } \\
\text { = mean time } \\
\text { udy; Time to } \\
\text { bolysis in M }\end{array}$ & $\begin{array}{l}\text { binant Tissue-Type } \\
\text { iation of treatment } \\
\text { ont = time from sy } \\
\text { I Infarction trial; Th }\end{array}$ & $\begin{array}{l}\text { lasminogen } \\
\text { til coronary } \\
\text { tom onset } \\
=\text { Tissue }\end{array}$ \\
\hline
\end{tabular}




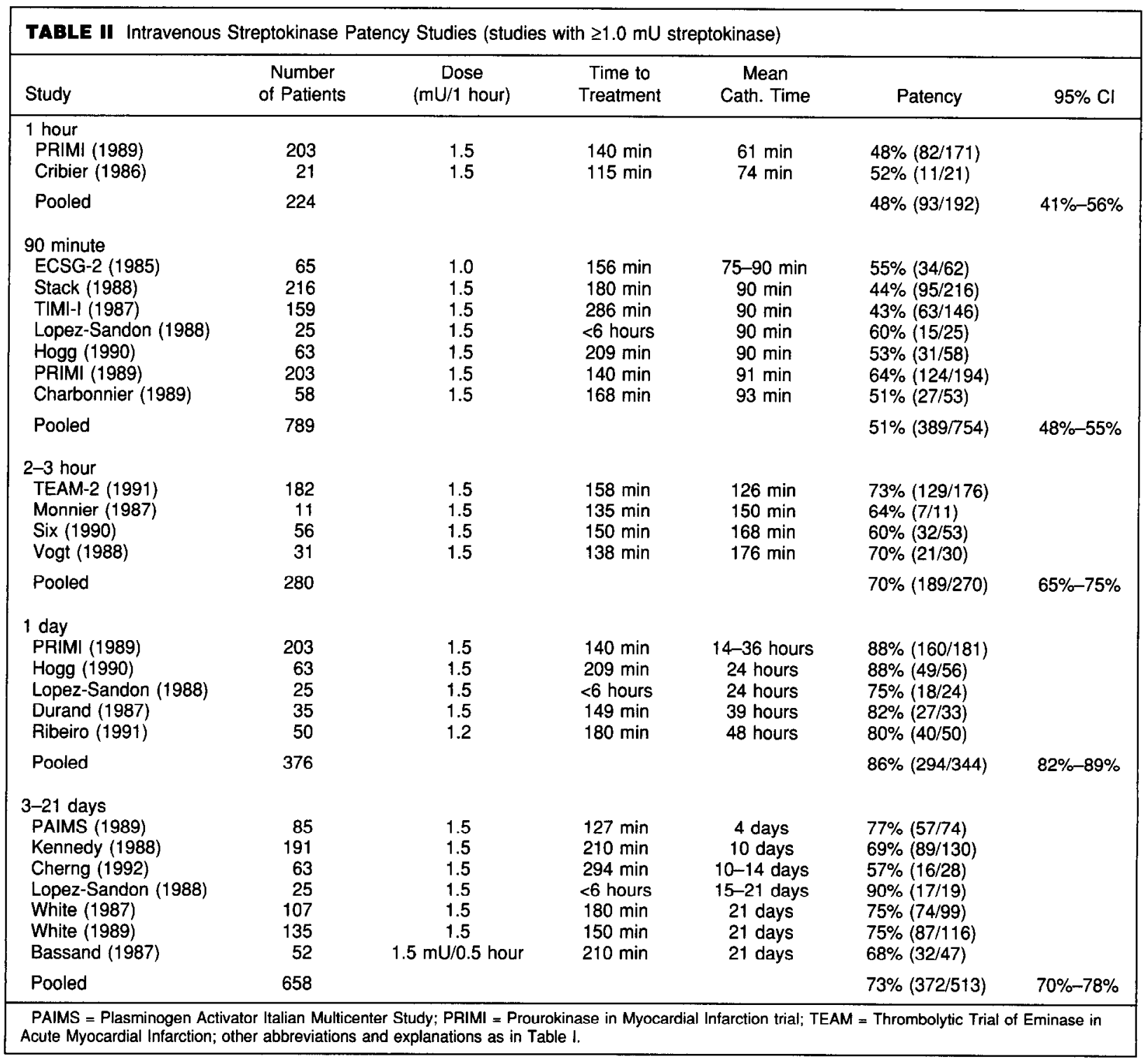

minations. A "weighted average" for the cjection fraction was determined for the thrombolytic and the control groups by adding the multiples of the mean ejection fraction and sample size for each study and dividing by the total sample size. The difference between the mean ejection fractions at each time point was determined.

Statistical analysis: For each point estimate of patency, 95\% confidence intervals were calculated. Because the time-patency curves for the different agents were generated from nonrandomized, distinct studies, formal statistical analyses for differences were not performed. The pooled rates of reocclusion were compared for statistical significance using a chi-square test. The difference between the thrombolytic-treated and control ejection fraction at each time point was tested with a Student's $t$ test.

\section{RESULTS}

Infarct-related artery patency: Rates of infarct-related artery patency at various times after initiation of thrombolytic regimens are listed in Tables I to V. Figure 1 illustrates the pooled results of the 14,124 angiographic observations. ${ }^{2-58}$ Early patency (60 and 90 minutes after initiation of thrombolytic therapy) is lowest with streptokinase, similar for t-PA and APSAC, and higher for accelerated-dose t-PA. By 2 to 3 hours, there is little difference in patency among standard t-PA-, streptokinase, and APSAC-treated patients. By 1 day and beyond, the patency rates are similar for all 4 regimens. In fact, by 2 to 3 weeks after presentation, the difference in patency between those treated with thrombolysis and those not treated is less striking, with approximately $60 \%$ of control patients or heparin-treated patients exhibiting infarct-related artery patency.

The percentage of initially treated patients included in the patency determination declined over time, so that $90 \%$ to $95 \%$ of patients were included in the 90 -minute time point and only $75 \%$ to $85 \%$ in the 3 - to 21 -day time point (Tables I to V).

Reocclusion: All studies included in the reocclusion analysis used intravenous heparin in conjuction with thrombolytic therapy, with the traditional dose of a 5,000 $\mathrm{U}$ bolus and 1,000 U/hour infusion.

Rates of reocclusion in trials randomizing to t-PA versus the nonfibrin-specific agents streptokinase, ${ }^{3}$ APSAC,${ }^{53}$ or urokinase ${ }^{40,42,45}$ are listed in Table VI. Re- 


\begin{tabular}{|c|c|c|c|c|c|c|}
\hline Study & $\begin{array}{l}\text { Number } \\
\text { of Patients }\end{array}$ & Dose & $\begin{array}{l}\text { Time to } \\
\text { Treatment }\end{array}$ & $\begin{array}{c}\text { Mean } \\
\text { Cath. Time }\end{array}$ & Patency & $95 \% \mathrm{Cl}$ \\
\hline \multicolumn{7}{|l|}{1 hour } \\
\hline ECSG-5 (1988) & 183 & $100 \mathrm{mg} / 3$ hours & $156 \mathrm{~min}$ & $42 \mathrm{~min}$ & $60 \%(108 / 180)$ & \\
\hline Smalling $(1990)$ & 91 & $1.25 \mathrm{mg} / \mathrm{kg} / 3$ hours & $228 \mathrm{~min}$ & $56 \mathrm{~min}$ & $45 \%(40 / 89)$ & \\
\hline RAAMI (1992) & 138 & $100 \mathrm{mg} / 3$ hours & $168 \mathrm{~min}$ & $62 \mathrm{~min}$ & $63 \%(54 / 86)$ & \\
\hline Topol (1987) & 75 & $1.25 \mathrm{mg} / \mathrm{kg} / 3$ hours & $216 \min$ & $68 \mathrm{~min}$ & $57 \%(40 / 70)$ & \\
\hline Pooled & 487 & & & & $57 \%(242 / 425)$ & $52 \%-61 \%$ \\
\hline \multicolumn{7}{|l|}{90 minute } \\
\hline ECSG-2 (1985) & 64 & $0.75 \mathrm{mg} / \mathrm{kg} / 90 \mathrm{~min}$ & $180 \mathrm{~min}$ & $75-90 \mathrm{~min}$ & $70 \%(43 / 61)$ & \\
\hline TIMI-IIA (1988) & 133 & $100 \mathrm{mg} / 6$ hours & $168 \mathrm{~min}$ & $84 \mathrm{~min}$ & $75 \%(98 / 131)$ & \\
\hline TIMI-I (1987) & 157 & $80 \mathrm{mg} / 3$ hours & $287 \min$ & $90 \mathrm{~min}$ & $70 \%(100 / 143)$ & \\
\hline RAAMI (1992) & 138 & $100 \mathrm{mg} / 3$ hours & $168 \min$ & $90 \mathrm{~min}$ & $77 \%(94 / 122)$ & \\
\hline ECSG-1 (1985) & 64 & $0.75 \mathrm{mg} / \mathrm{kg} / 90 \mathrm{~min}$ & $204 \mathrm{~min}$ & $90 \mathrm{~min}$ & $61 \%(38 / 62)$ & \\
\hline TAMI-4 (1989) & 50 & $100 \mathrm{mg} / 3$ hours & $243 \min$ & $90 \mathrm{~min}$ & $52 \%(26 / 50)$ & \\
\hline TAMI-5 (1991) & 95 & $100 \mathrm{mg} / 3$ hours & $200 \mathrm{~min}$ & $90 \mathrm{~min}$ & $71 \%(67 / 95)$ & \\
\hline Topol (1987) & 75 & $1.25 \mathrm{mg} / \mathrm{kg} / 3$ hours & $216 \min$ & $90 \mathrm{~min}$ & $69 \%(49 / 71)$ & \\
\hline Topol (1987) & 142 & $1 \mathrm{mg} / \mathrm{kg} /$ hour & $190 \mathrm{~min}$ & $90 \mathrm{~min}$ & $72 \%(102 / 142)$ & \\
\hline Johns (1988) & 68 & $1 \mathrm{mg} / \mathrm{kg} / 90 \mathrm{~min}$ & $180 \mathrm{~min}$ & $90 \mathrm{~min}$ & $76 \%(52 / 68)$ & \\
\hline CRAFT (1991) & 206 & $100 \mathrm{mg} / 3$ hours & $<6$ hours & $90 \mathrm{~min}$ & $63 \%(126 / 199)$ & \\
\hline Smalling (1990) & 91 & $1.25 \mathrm{mg} / \mathrm{kg} / 3$ hours & $228 \mathrm{~min}$ & $90 \mathrm{~min}$ & $70 \%(61 / 87)$ & \\
\hline TAMI-3 (1989) & 134 & $1.5 \mathrm{mg} / \mathrm{kg} / 4$ hours & $168 \mathrm{~min}$ & $92 \mathrm{~min}$ & $79 \%(104 / 131)$ & \\
\hline KAMIT (1991) & 107 & $100 \mathrm{mg} / 3$ hours & $180 \mathrm{~min}$ & $95 \min$ & $64 \%(65 / 102)$ & \\
\hline GAUS (1988) & 124 & $70 \mathrm{mg} / 90 \mathrm{~min}$ & $<4$ hours & $97 \mathrm{~min}$ & $69 \%(84 / 121)$ & \\
\hline Pooled & 1,648 & & & & $70 \%(1,109 / 1,585)$ & $68 \%-72 \%$ \\
\hline \multicolumn{7}{|l|}{$2-3$ hour } \\
\hline Topol (1987) & 75 & $1.25 \mathrm{mg} / \mathrm{kg} / 3$ hours & $216 \mathrm{~min}$ & $120 \mathrm{~min}$ & $79 \%(59 / 75)$ & \\
\hline Guerci (1987) & 72 & $80 / 100 \mathrm{mg} / 3$ hours & $192 \mathrm{~min}$ & $150 \mathrm{~min}$ & $66 \%(44 / 67)$ & \\
\hline Pooled & 147 & & & & $73 \%(103 / 142)$ & $65 \%-80 \%$ \\
\hline \multicolumn{7}{|l|}{1 day } \\
\hline GAUS (1988) & 124 & $70 \mathrm{mg} / 90 \mathrm{~min}$ & $<4$ hours & 24 hours & $78 \%(82 / 105)$ & \\
\hline TEAM-3 (1992) & 164 & $100 \mathrm{mg}$ & $168 \mathrm{~min}$ & 33 hours & $86 \%(128 / 149)$ & \\
\hline TIMI-IIA (1988) & 128 & $100 / 150 \mathrm{mg} / 6$ hours & 174 min & 33 hours & $82 \%(93 / 114)$ & \\
\hline TIMI-II (1989) & 1,366 & $100 \mathrm{mg}$ & $156 \min$ & 33 hours & $85 \%(1,040 / 1,229)$ & \\
\hline Pooled & 1,782 & & & & $84 \%(1,343 / 1,597)$ & $82 \%-86 \%$ \\
\hline \multicolumn{7}{|l|}{$3-21$ days } \\
\hline ECSG-6 (1992) & 652 & $100 \mathrm{mg} / 3$ hours & $170 \mathrm{~min}$ & 3.4 days & $79 \%(410 / 518)$ & \\
\hline PAIMS (1989) & 86 & $100 \mathrm{mg} / 3$ hours & $124 \mathrm{~min}$ & 4.1 days & $81 \%(63 / 78)$ & \\
\hline Bassand (1989) & 93 & $100 \mathrm{mg} / 3$ hours & $172 \min$ & 5.4 days & $76 \%(64 / 84)$ & \\
\hline NHFA (1988) & 73 & $100 \mathrm{mg} / 3$ hours & $195 \mathrm{~min}$ & 5-7 days & $70 \%(43 / 61)$ & \\
\hline TIMI-HA (1988) & 389 & $100 / 150 \mathrm{mg} / 6$ hours & $174 \min$ & $7-10$ days & $79 \%(240 / 303)$ & \\
\hline Thompson (1991) & 241 & $100 \mathrm{mg} / 3$ hours & $155 \min$ & $7-10$ days & $80 \%(157 / 196)$ & \\
\hline GAUS (1988) & 124 & $70 \mathrm{mg} / 3$ hours & $<4$ hours & 10 days & $73 \%(63 / 86)$ & \\
\hline Cherng (1992) & 59 & $100 \mathrm{mg} / 3$ hours & $312 \mathrm{~min}$ & 10-14 days & $77 \%(23 / 30)$ & \\
\hline Rapold (1989) & 34 & $100 \mathrm{mg} / 3$ hours & $186 \min$ & 12.5 days & $81 \%(25 / 31)$ & \\
\hline ECSG-5 (1988) & 367 & $100 \mathrm{mg} / 3$ hours & $156 \mathrm{~min}$ & 15 days & $87 \%(283 / 327)$ & \\
\hline White (1989) & 135 & $100 \mathrm{mg} / 3$ hours & $150 \mathrm{~min}$ & 21 days & $76 \%(94 / 124)$ & \\
\hline O'Rourke (1988) & 74 & $100 \mathrm{mg} / 3$ hours & $120 \mathrm{~min}$ & 21 days & $81 \%(55 / 68)$ & \\
\hline Pooled & 2,327 & & & & $80 \%(1,520 / 1,906)$ & $78 \%-81 \%$ \\
\hline
\end{tabular}

occlusion occurred nearly twice as often with t-PA than with nonfibrin-specific thrombolytic agents $(p=0.002)$.

Left ventricular ejection fraction: Studies evaluating LV ejection fraction, by contrast or radionuclide angiography, after randomizing patients to thrombolytic therapy versus control groups are listed in Table VII. $9,10,12-17,59,60$ The difference in ejection fraction between thrombolytic and control groups as a function of time after treatment is illustrated in Figure 2. Patients treated with thrombolysis had higher ejection fractions than control patients, although the absolute difference was only $3 \%$ at 10 to 28 days after treatment.

\section{DISCussion}

Infarct-related artery patency: This analysis confirms previous findings that early patency rates were higher with t-PA and APSAC than with streptokinase, and that the highest early rates of patency were with accelerated-dose t-PA. By 2 to 3 hours, the patency rates of streptokinase, standard dose t-PA, and APSAC were 


\begin{tabular}{|c|c|c|c|c|c|c|}
\hline Study & $\begin{array}{l}\text { Number } \\
\text { of Patients }\end{array}$ & Dose & $\begin{array}{l}\text { Time to } \\
\text { Treatment }\end{array}$ & $\begin{array}{c}\text { Mean } \\
\text { Cath. Time }\end{array}$ & Patency & $95 \% \mathrm{Cl}$ \\
\hline $\begin{array}{l}1 \text { hour } \\
\text { Gemmell (1991) } \\
\text { Smalling (1990) } \\
\text { Neuhaus (1989) } \\
\text { TIMI-IIP (1987) } \\
\text { Purvis (1991) } \\
\text { TAPS (1992) } \\
\text { RAAMI (1991) }\end{array}$ & $\begin{array}{r}33 \\
84 \\
80 \\
33 \\
60 \\
210 \\
143\end{array}$ & $\begin{array}{c}70 \mathrm{mg} / 1 \text { hour } \\
1.2 \mathrm{mg} / \mathrm{kg} / 60 \mathrm{~min} \\
100 \mathrm{mg} / 90 \mathrm{~min} \\
90 \mathrm{mg} / 60 \mathrm{~min} \\
70-100 \mathrm{mg} / 60 \mathrm{~min} \\
100 \mathrm{mg} / 90 \mathrm{~min} \\
100 \mathrm{mg} / 90 \mathrm{~min}\end{array}$ & $\begin{array}{l}208 \mathrm{~min} \\
216 \mathrm{~min} \\
<6 \text { hours } \\
180 \mathrm{~min} \\
130 \mathrm{~min} \\
162 \mathrm{~min} \\
162 \mathrm{~min}\end{array}$ & $\begin{array}{l}49 \mathrm{~min} \\
56 \mathrm{~min} \\
60 \mathrm{~min} \\
60 \mathrm{~min} \\
60 \mathrm{~min} \\
61 \mathrm{~min} \\
61 \mathrm{~min}\end{array}$ & $\begin{array}{l}77 \%(23 / 30) \\
65 \%(51 / 79) \\
74 \%(54 / 73) \\
82 \%(27 / 33) \\
80 \%(35 / 44) \\
73 \%(146 / 199) \\
76 \%(66 / 87)\end{array}$ & \\
\hline Pooled & 643 & & & & $74 \%(402 / 545)$ & $70 \%-77 \%$ \\
\hline $\begin{array}{l}90 \text { minute } \\
\text { Neuhaus (1989) } \\
\text { Smalling (1990) } \\
\text { TAMI-7 (1992) } \\
\text { Gemmell (1991) } \\
\text { Purvis (1991) } \\
\text { RAAMI (1992) } \\
\text { TAPS (1992) }\end{array}$ & $\begin{array}{r}80 \\
84 \\
61 \\
33 \\
60 \\
143 \\
210\end{array}$ & $\begin{array}{c}100 \mathrm{mg} / 90 \mathrm{~min} \\
1.2 \mathrm{mg} / \mathrm{kg} / 60 \mathrm{~min} \\
1.25 \mathrm{mg} / \mathrm{kg} / 90 \mathrm{~min} \\
70 \mathrm{mg} / 1 \mathrm{hour} \\
70-100 \mathrm{mg} / 60 \mathrm{~min} \\
100 \mathrm{mg} / 90 \mathrm{~min} \\
100 \mathrm{mg} / 90 \mathrm{~min}\end{array}$ & $\begin{array}{l}<6 \text { hours } \\
216 \mathrm{~min} \\
151 \mathrm{~min} \\
208 \mathrm{~min} \\
130 \mathrm{~min} \\
162 \mathrm{~min} \\
162 \mathrm{~min}\end{array}$ & $\begin{array}{l}90 \mathrm{~min} \\
90 \mathrm{~min} \\
90 \mathrm{~min} \\
90 \mathrm{~min} \\
90 \mathrm{~min} \\
91 \mathrm{~min} \\
91 \mathrm{~min}\end{array}$ & $\begin{array}{l}91 \%(67 / 74) \\
84 \%(68 / 81) \\
84 \%(51 / 61) \\
87 \%(26 / 30) \\
81 \%(48 / 59) \\
81 \%(104 / 128) \\
84 \%(168 / 199)\end{array}$ & \\
\hline Pooled & 671 & & & & $84 \%(532 / 632)$ & $82 \%-87 \%$ \\
\hline $\begin{array}{l}1 \text { day } \\
\text { Neuhaus (1989) } \\
\text { Gemmell (1991) } \\
\text { TAPS (1992) }\end{array}$ & $\begin{array}{r}80 \\
33 \\
210\end{array}$ & $\begin{array}{c}100 \mathrm{mg} / 90 \mathrm{~min} \\
70 \mathrm{mg} / 1 \mathrm{hour} \\
100 \mathrm{mg} / 90 \mathrm{~min}\end{array}$ & $\begin{array}{l}<6 \text { hours } \\
208 \mathrm{~min} \\
162 \mathrm{~min}\end{array}$ & $\begin{array}{c}24 \text { hours } \\
24 \text { hours } \\
24-48 \text { hours }\end{array}$ & $\begin{array}{l}92 \%(61 / 66) \\
83 \%(24 / 29) \\
85 \%(168 / 198)\end{array}$ & \\
\hline Pooled & 323 & & & & $86 \%(253 / 293)$ & $82 \%-90 \%$ \\
\hline $\begin{array}{l}3-21 \text { days } \\
\text { TAPS (1992) }\end{array}$ & 210 & $100 \mathrm{mg} / 90 \mathrm{~min}$ & $162 \mathrm{~min}$ & 14-21 days & $89 \%(157 / 177)$ & $85 \%-94 \%$ \\
\hline
\end{tabular}

similar, a finding that persisted at 24 hours and at 5 to 7 days. By 10 to 21 days, the patency rates even without thrombolytic treatment were surprisingly high-61\% (95\% confidence interval $57 \%$ to $64 \%$ ). These findings are consistent with the GUSTO angiographic substudy results, ${ }^{1}$ which found higher patency rates with accelerated t-PA than streptokinase at 90 minutes, but no significant difference in patency at 3 hours, 24 hours, or 7 days.

Assessment of patency at later time points may be affected by patient dropout. By 1 day after treatment, typically only $85 \%$ to $90 \%$ of patients originally enrolled in a study will have analyzable angiographic data, and at later times only about $80 \%$ (data contained in Tables I to V). Because the patients who withdraw are more unstable or may have died, and because such patients are more likely to have occluded infarct-related arteries than the surviving patients, ${ }^{61}$ the reported patency rates may overestimate the true rate.

Reocclusion: In assessing reocclusion, comparison of agents between different nonrandomized studies should be regarded with caution because of the lack of a common definition of reocclusion. For this analysis, reocclusion was defined as angiographically documented occlusion of the infarct artery that had been patent either

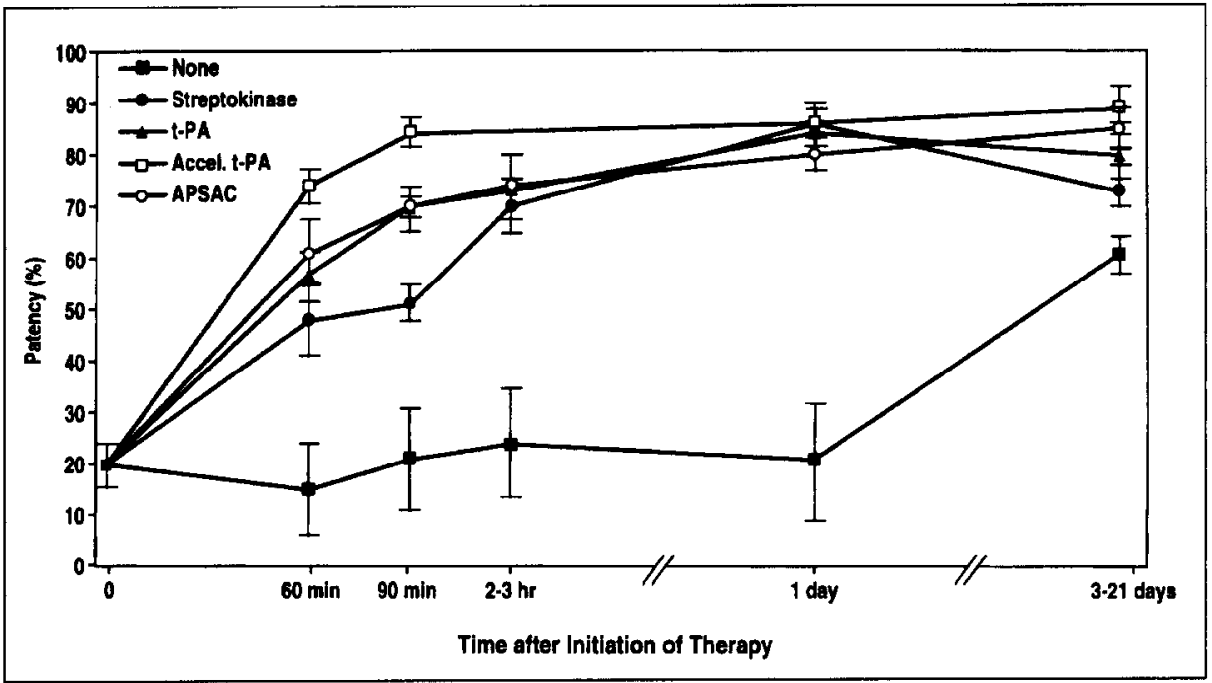

FIUURE 1. Pooled angographic patency rates, with $95 \%$ confidence intervals, over time after no thrombolytic agent, streptokr nase, conventional dose tiscue-type plasminogen activator (t-PA), accelerated (Accel.) dose t-PA, and anisoylated plasminogen streptokinase activator complex (APSAC). Rates include 14,124 anglographIc observations. 


\begin{tabular}{|c|c|c|c|c|c|c|}
\hline Study & $\begin{array}{l}\text { Number } \\
\text { of Patients }\end{array}$ & Dose & $\begin{array}{l}\text { Time to } \\
\text { Treatment }\end{array}$ & $\begin{array}{c}\text { Mean } \\
\text { Cath. Time }\end{array}$ & Patency & $95 \% \mathrm{Cl}$ \\
\hline $\begin{array}{l}1 \text { hour } \\
\text { TAPS (1992) } \\
\text { Kasper (1986) }\end{array}$ & $\begin{array}{r}210 \\
50\end{array}$ & $\begin{array}{l}30 \mathrm{U} \\
30 \mathrm{U}\end{array}$ & $\begin{array}{l}150 \mathrm{~min} \\
151 \mathrm{~min}\end{array}$ & $\begin{array}{l}60 \mathrm{~min} \\
64 \mathrm{~min}\end{array}$ & $\begin{array}{l}60 \%(123 / 204) \\
64 \%(32 / 50)\end{array}$ & \\
\hline Pooled & 260 & & & & $61 \%(155 / 254)$ & $55 \%-67 \%$ \\
\hline $\begin{array}{l}90 \text { minute } \\
\text { TAPS (1992) } \\
\text { Lopez-Sendon (1988) } \\
\text { Hogg (1990) } \\
\text { Charbonnier (1989) } \\
\text { Relik-van Wely (1991) }\end{array}$ & $\begin{array}{r}210 \\
22 \\
65 \\
58 \\
156\end{array}$ & $\begin{array}{l}30 \mathrm{U} \\
30 \mathrm{U} \\
30 \mathrm{U} \\
30 \mathrm{U} \\
30 \mathrm{U}\end{array}$ & $\begin{array}{l}150 \text { min } \\
<6 \text { hours } \\
199 \min \\
162 \min \\
106 \min \end{array}$ & $\begin{array}{l}90 \mathrm{~min} \\
90 \mathrm{~min} \\
90 \mathrm{~min} \\
95 \mathrm{~min} \\
96 \mathrm{~min}\end{array}$ & $\begin{array}{l}70 \%(142 / 202) \\
86 \%(19 / 22) \\
53 \%(32 / 60) \\
70 \%(38 / 54) \\
73 \%(106 / 145)\end{array}$ & \\
\hline Pooled & 511 & & & & $70 \%(337 / 483)$ & $66 \%-74 \%$ \\
\hline $\begin{array}{l}\text { 2-3 hours } \\
\text { TEAM-2 (1991) } \\
\text { Vogt }(1988) \\
\text { Monnier (1987) }\end{array}$ & $\begin{array}{r}188 \\
30 \\
14\end{array}$ & $\begin{array}{l}30 \mathrm{U} \\
30 \mathrm{U} \\
30 \mathrm{U}\end{array}$ & $\begin{array}{l}159 \min \\
142 \min \\
141 \min \end{array}$ & $\begin{array}{l}2.1 \text { hours } \\
2.6 \text { hours } \\
2.7 \text { hours }\end{array}$ & $\begin{array}{l}72 \%(132 / 183) \\
77 \%(23 / 30) \\
93 \%(13 / 14)\end{array}$ & \\
\hline Pooled & 232 & & & & $74 \%(168 / 227)$ & $68 \%-80 \%$ \\
\hline $\begin{array}{l}1 \text { day } \\
\text { Hogg (1990) } \\
\text { Lopez-Sendon (1988) } \\
\text { TEAM-3 (1992) } \\
\text { TAPS (1992) } \\
\text { SWIFT (1991) }\end{array}$ & $\begin{array}{r}65 \\
22 \\
161 \\
210 \\
397\end{array}$ & $\begin{array}{l}30 \mathrm{U} \\
30 \mathrm{U} \\
30 \mathrm{U} \\
30 \mathrm{U} \\
30 \mathrm{U}\end{array}$ & $\begin{array}{l}199 \min \\
<6 \text { hours } \\
156 \mathrm{~min} \\
150 \mathrm{~min} \\
180 \mathrm{~min}\end{array}$ & $\begin{array}{l}24 \text { hours } \\
24 \text { hours } \\
30 \text { hours } \\
24-48 \text { hours } \\
48 \text { hours }\end{array}$ & $\begin{array}{l}81 \%(47 / 58) \\
91 \%(20 / 22) \\
89 \%(133 / 149) \\
93 \%(183 / 196) \\
68 \%(255 / 373)\end{array}$ & \\
\hline Pooled & 855 & & & & $80 \%(638 / 798)$ & $77 \%-83 \%$ \\
\hline $\begin{array}{l}\text { 3-21 days } \\
\text { Bassand (1989) } \\
\text { TAPS }(1992) \\
\text { Lopez-Sendon (1988) }\end{array}$ & $\begin{array}{r}112 \\
210 \\
22\end{array}$ & $\begin{array}{l}30 \mathrm{U} \\
30 \mathrm{U} \\
30 \mathrm{U}\end{array}$ & $\begin{array}{l}187 \text { min } \\
150 \text { min } \\
<6 \text { hours }\end{array}$ & $\begin{array}{c}3.9 \text { days } \\
14-21 \text { days } \\
15-21 \text { days }\end{array}$ & $\begin{array}{l}77 \%(82 / 106) \\
90 \%(157 / 174) \\
81 \%(17 / 21)\end{array}$ & \\
\hline Pooled & 344 & & & & $85 \%(256 / 301)$ & $81 \%-89 \%$ \\
\hline
\end{tabular}

initially or after an intervention on a 90-minute angiogram. Because patients who had patent infarct-related arteries at initial angiography and then died or had reinfarction without follow-up angiography are not included as reoccluded, the reocclusion rates reported likely underestimate the true rates.

This analysis included only studies in which patients were randomized between t-PA and a nonfibrin-specific agent (either streptokinase, APSAC, or urokinase), which results in a more pronounced systemic fibrinolytic state that theoretically reduces the risk of reocclusion. Despite the use of intravenous heparin, which improves infarct-related artery patency after t-PA, ${ }^{47,62}$ t-PA was associated with a substantially higher rate of reocclusion than the nonfibrin-specific agents. The reocclusion results are in contrast to the GUSTO angiographic substudy results, ${ }^{1}$ where the rate of reocclusion was low with both accelerated t-PA and streptokinase. In the
FIGURE 2. Difference in left ventricular ejection fraction (EF) between thrombolytictreated and control patients versus time after thrombolytic treatment. Data are from randomized trials of thrombolytic therapy versus control, and are pooled at the serial time points. Data include 3,066 ventriculographic observa tions.

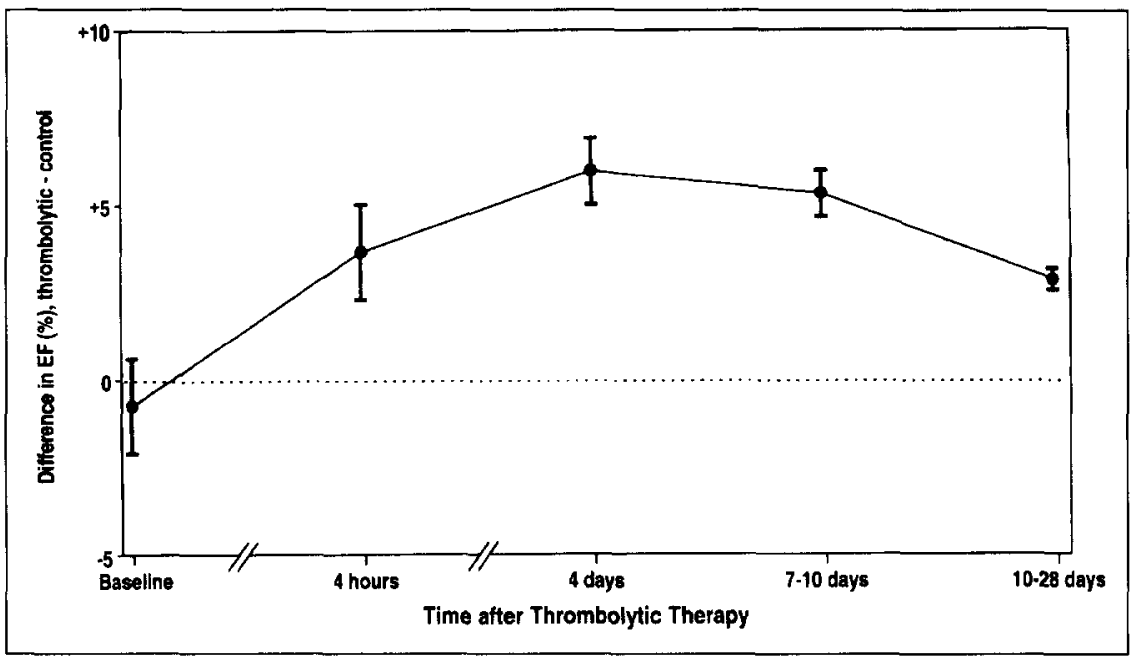




\begin{tabular}{|c|c|c|c|c|}
\hline \multirow[b]{2}{*}{ Study } & \multicolumn{2}{|c|}{ Timing of Catheterization } & \multicolumn{2}{|c|}{ Thrombolytic Agent } \\
\hline & Early Cath. & Late Cath. & $t-P A$ & SK, APSAC or UK \\
\hline $\begin{array}{l}\text { TIMI-I (1987) } \\
\text { TAPS (1992) } \\
\text { GAUS }(1988) \\
\text { CRAFT }(1991) \\
\text { TAMI-5 (1991) }\end{array}$ & $\begin{array}{l}90 \min \\
90 \mathrm{~min} \\
90 \mathrm{~min} \\
90 \mathrm{~min} \\
90 \mathrm{~min}\end{array}$ & $\begin{array}{l}\text { Predischarge } \\
24-48 \text { hours } \\
24 \text { hours } \\
7-10 \text { days } \\
5-10 \text { days }\end{array}$ & $\begin{array}{l}20 \%(17 / 86) \\
10 \%(18 / 179) \\
15 \%(13 / 88) \\
14 \%(26 / 180) \\
11 \%(10 / 94)\end{array}$ & $\begin{array}{c}9 \%(4 / 44) \text { (SK) } \\
2.4 \%(4 / 168) \text { (APSAC) } \\
6.5 \%(5 / 77) \text { (UK) } \\
15 \%(25 / 170) \text { (UK) } \\
6 \%(6 / 97) \text { (UK) }\end{array}$ \\
\hline Pooled & & & $\begin{array}{c}13.4 \%(84 / 627) \\
(95 \% \mathrm{Cl}: 11 \%-16 \%)\end{array}$ & $\begin{array}{l}8.0 \%(44 / 556) \\
(95 \% \mathrm{Cl}: 3 \%-10 \%)\end{array}$ \\
\hline
\end{tabular}

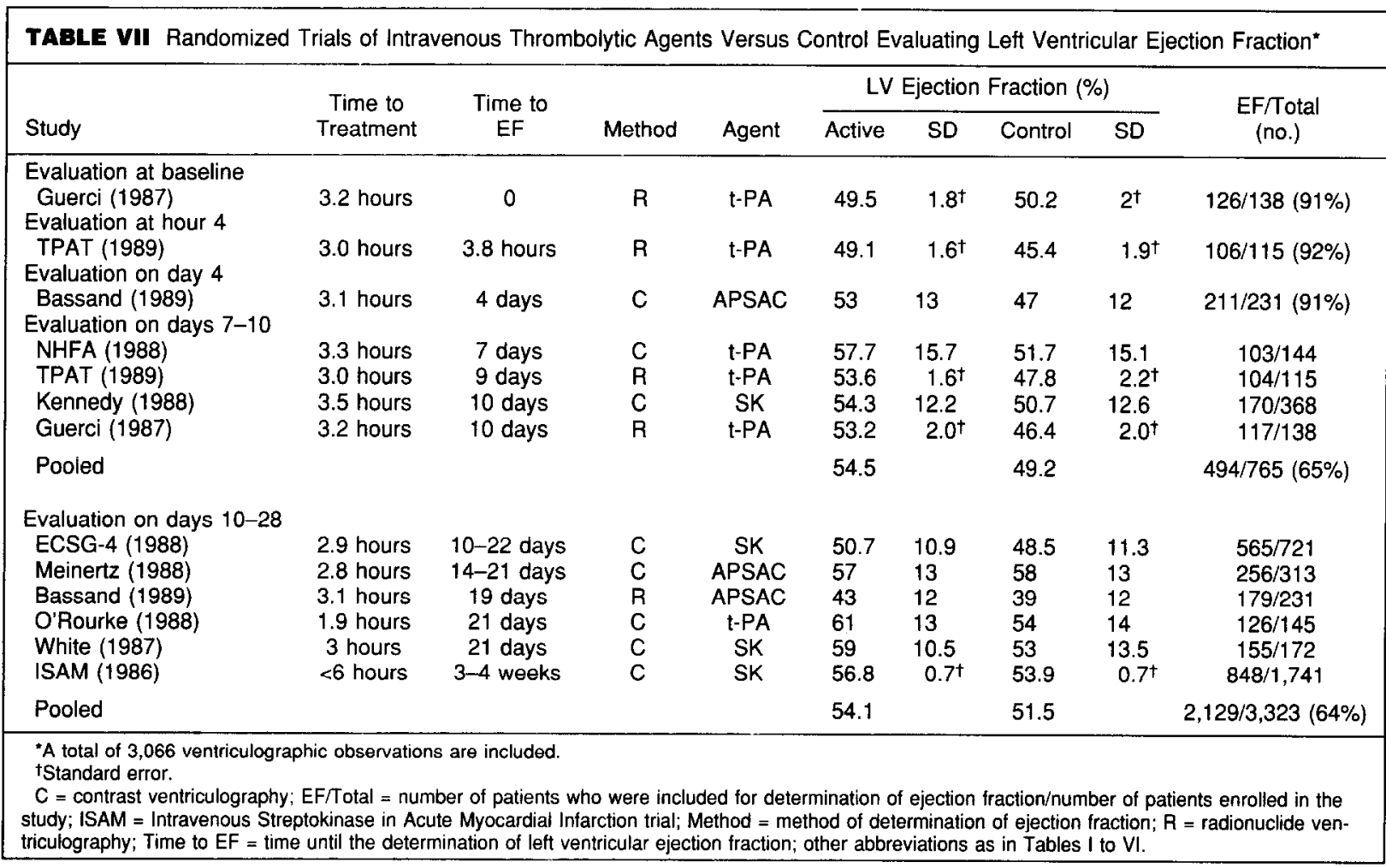

GUSTO trial, the heparin dose and adjustment scheme were more aggressive, with a target activated partial thromboplastin time of $\geq 2$ times control.

Left ventricular ejection fraction: Studies evaluating LV ejection fraction after intravenous streptokinase, t-PA, or APSAC versus control or conventional treatment have found a modest improvement in treated patients (Table VII). Contrary to theoretical evidence that myocardial function may not fully recover for several days or weeks after a severe ischemic insult, ${ }^{63}$ this analysis showed no further improvement in LV ejection fraction after day 4 evaluation in patients treated with thrombolytic therapy (Figure 2). This finding is consistent with an analysis of patients in the Thrombolysis and Angioplasty in Myocardial Infarction trials who underwent serial angiography after thrombolytic therapy. ${ }^{64}$ In contrast, patients not given thrombolytic therapy showed a late improvement in LV ejection fraction. This may be related to further reocclusion in treated patients and spontaneous reperfusion of initially collateralized zones in untreated patients. ${ }^{65}$

Studies using ejection fraction as an end point have been limited by a high proportion of missing values due to the typical $5 \%$ to $10 \%$ early mortality and additional $15 \%$ to $30 \%$ technically inadequate or unobtained studies. Because patients with missing values are those who have died and those who are sicker, a group with poor LV function, such studies generally overestimate true ventricular function. ${ }^{66}$ When patients with poor LV function who might otherwise have died are saved by thrombolytic therapy, factoring in their low ejection fractions decreases the ejection fraction in the thrombolytic-treated groups.

This pooled analysis, by incorporating all available data from studies that are individually too small to give stable estimates of angiographic end points, provides a context in which to interpret future angiographic reperfusion studies. 
1. The GUSTO Angiographic Investigators. The effects of tissue plasminogen activator, streptokinase, or both on coronary-artery patency, ventricular function, and survival after acute myocardial infarction. N Engl J Med 1993:329:1615-1622. 2. Anderson JL, Marshall HW, Askins JC, Lutz JR, Frederick PR, Sorenson SG, Menlove RL, Yanowitz FG, Hagan AD. A randomized trial of intravenous and intıacoronary streptokinase in patients with acute myocardial infarction. Circulation 1984;70:606-618.

3. Chesebro JH, Knatterud G, Roberts R, Borer J, Cohen LS, Dalen J, Dodge HT, Francis CK, Hillis D, Ludbrook P, Markis JE, Mueller H, Passamani ER, Powers ER, Rao AK, Robertson T, Ross $\Lambda$, Ryan TJ, Sobel BE, Willerson J, Williams DO, Zaret BL, Braunwald E. Thrombolysis in Myocardial Infarction (TIMI) trial, phase I: a comparison between intravenous tissue plasminogen activator and intravenous streptokinase. Circulation 1987;76:142-154.

4. Timmis AD, Griffin B, Crick J, Sowton E. Anisoylated plasminogen streptokinase activator complex in acute myocardial infarction: a placebo-controlled arteriographic coronary recanalization study. I Am Coll Cardiol 1987; 10:205-210. 5. Collen D, Topol EJ, Tiefenbrunn AJ, Gold HK, Weisfeldt ML, Sobel BE, Leinbach RC, Brinker JA, Ludbrook PA, Yasuda I, Bulkley BH, Robison AK, Hutter AM, Bell WR, Spadaro JJ, Khaw BA, Grossbard EB. Coronary thrombolysis with recombinant human tissue-type plasminogen activator: a prospective, randomized, placebo-controlled trial. Circulation 1984;70:1012-1017.

6. Topol EJ, Bates ER, Walton JA, Baumann G, Wolfe S, Maino J, Bayer L, Gorman L, Kline E, O'Neill W, Pitt B. Community hospital administration of intravenous tissue plasminogen activator in acute myocardial infarction: improved timing, thrombolytic efficacy and ventricular function. I Am Coll Cardiol 1987;10:1173-1177. 7. Cribier A, Berland J, Saoudi N, Redonnet M, Moore N, Letac B. Intracoronary streptokinase, OK!... intravenous streptokinase, first? Heparin or intravenous streptokinase in acute infarction: preliminary results of a prospective randomized trial with angiographic evaluation in 44 patients. Haemostasis 1986;16:122-129. 8. Verstraete M, Bemard R, Brower RW, Collen D. Dunning AJ, Lubsen J, Mathey DG. Double-blind randomised trial of intravenous tissue-type plasminogen activator versus placebo in acute myocardial infarction (ECSG-1). Lancet 1985:2:965-696. 9. Guerci AD, Gerstenblith G, Brinker JA, Chandra NC, Gottlieb SO, Bahr RD Weiss JL, Shapiro EP, Flaherty JT, Bush DE, Chew PH, Gottlieb SH, Halperin HR, Ouyag P, Walford GD, Bell WR, Fatterpaker AK, Llewellyn M, Topol EJ, Healy B, Siu CO, Becker LC, Weisfeldt ML. A randuntized trial of intravenous tissue plasminogen activator for acute myocardial infarction with subsequent randomization to elective coronary angioplasty. $N$ Engl J Med 1987;317:1613-1618.

10. Armstrong PW, Baigrie RS, Daly PA, Haq A, Gent M, Roberts R, Freeman M, Burns R, Liu P. Morgan C. Tissue plasminogen activator: Toronto (TPAT). Placebo-controlled randomized trial in acute myocardial infarction. $J \mathrm{Am} \mathrm{Coll} \mathrm{Car}$ diol 1989;13:1469-1476

11. Durand $P$, Asseman $P$, Pruvost $P$, Bertrand ME, LaBlanche JM, Thery C. Effectiveness of intravenous streptokinase on infarct size and $\mathrm{LV}$ function in acute myocardial infarction. Clin Cardiol 1987:10:383-392.

12. Bassand JP, Machecoert J, Cassagnes J, Anguenot T, Luson R, Borel E. Peycelon P, Wolf E, Ducellier D, for the APSIM Study Investigators. Multicenter trial of intravenous anisoylated plasminogen streptokinase activator complex (APSAC) in acute myocardial infarction: effects on infarct size and left ventricular function. J Am Coll Cardiol 1989;13:988-997.

13. National Heart Foundation of Australia Coronary Thrombolysis Group. Coronary thrombolysis and myocardial salvage by tissuc plasminogen activator given up to 4 hours after onset of myocardial infarction. Lancet 1988;2:203-208.

14. Kennedy JW, Martin GV, Davis KB, Maynard C, Stadius M, Sheehan FH, Ritchie JL. The Western Washington intravenous streptokinase in acute myocardial infarction randomized trial. Circulation 1988;77:345-352.

15. Van de Werf $F$, Arnold $A$. Intravenous tissue plasminogen activator and size of infarct, left ventricular function, and survival in acute myocardial infarction (ECSG-4). Br Med J 1988;297:1374-1379.

16. White HD, Norris RM, Brown MA, Takayama M, Maslowski A, Bass N, Ormiston J, Whitlock T. Effect of intravenous streptokinase on left ventricular function and early survival after acute myocardial infarction. N Engl J Med 1987:317:850-855. 17. O'Rourke M, Baron D, Keogh A, Kelly R, Nelson G, Barnes C, Raftos J, Graham K, Hillman K, Newman H, Healey J, Woolridge J, Rivers J, White H, Whitlock R, Norris R. Limitation of myocardial infarction by early infusion of recombinant tissue-type plasminogen activator. Circulation 1988;77:1311-1315.

18. Bassand JP, Faivre R, Becque O, Habert C, Schuffenecher M, Petiteau P, Cardot JC, Verdenet J, LaRoze M, Maurat JP. Effects of early high-dose streptokinase intravenously on left ventricular function in acute myocardial infarction. Am J Cardiol $1987: 60: 435-439$

19. PRIMI Trial Study Group. Randomized double blind trial of recombinant prourokinase against streptokinase in acute myocardial infarction. Lancet 1989;1: 863-868.

20. Verstraete M, Bory M, Collen D, Erbel R, Lennane RJ, Lubsen J. Randomized trial of intravenous recombinant tissue-type plasminogen activator versus intravenous streptokinase in acute myocardial infarction (ECSG-2). Lancet 1985;1: $842-847$.

21. Stack RS, O'Connor CM, Mark DB, Hinohara T, Phillips HR, Lee MM Ramirez NM, O'Callaghan WG, Simonton CA, Carlson EB, Morris KG, Behar VS, Kong Y, Peter RH, Califf RM. Coronary perfusion during acute myocardial infarction with a combined therapy of coronary angioplasty and high-dose intravenous streptokinase. Circulation 1988:77:151-161.

22. Lopez-Sendon J, Seabra-Gomes R, Macaya C, Santos FM, Munoz J, Sobrino N, Calvo L, Silva J, Miguel J, Lopez de Sa E, Jadrague LM, La Paz H. Intravenous anisoylated plasminogen streptokinase activator complex versus intravenous streptokinase in myocardial infarction. A randomized multicenter study (abstr). Circulation 1988;78(suppl II):II-277.

23. Hogg KJ, Gemmill JD, Burns J, Litson WK, Rae AP, Dunn FG, Hillis WS Angiographic patency study of APSAC versus streptokinase in acute myocardial infarction. Lancet 1990;335:254-258.

24. Charbonnier B, Cribier A, Monassier JP, Favier JP, Materne P, Brochier ML, Letac B, Hanssen M, Sacrez A, Kulbertus H. Etude Eurpéenne multicentrique et randomisée de l'APSAC versus streptokinase dans l'infarctus du myocarde. Arch Mal Cocur Vaiss 1989;82:1565 1571.

25. Anderson JL, Sorenson S, Moreno F, Hackworthy R, Browne K, Dale HT, Leya F, Dangoisse V, Eckerson H, Marder V, for the TEAM-2 Study Investigators. Multicenter patency trial of intravenous APSAC compared with streptokinase in acute myocardial infarction. Circulation 1991;88:126-140.

26. Monnier P, Sigwart U, Vincent A, Bachmann F, Goy JJ, Schaller MD, Kaufman U, Badan M, Grbic M, Perret C. Anisoylated plasminogen streptokinase activator complex versus streptokinase in acute myocardial infarction. Drugs 1987;33 (suppl 3): 175-178.

27. Six AJ, Louwerenburg HW, Braams R, Mechelse K, Mosterd WL, Bredero $\mathrm{AC}$, Dunselman P, van Hemel N. A double-blind randomized multicenter doseranging trial of intravenous streptokinase in acute myocardial infarction. Am J Cardiol 1990;65:119-123.

28. Vogt P, Schaller MD, Monnicr P, Kaufmann U, Goy JJ. Systemic thrombolysis in acute myocardial infarction: bolus injection of APSAC versus infusion of streptokinase (abstr). Eur Heart J 1988;9(suppl A):213.

29. Ribeiro EE, Silva LA, Cameiro R, D'Oliveira LG, Gasques A, Amino JG, Tavares JR, Torossian S, Buffolo E, Fo RD. A randomized trial of direct PTCA vs intravenous streptokinase in acute myocardial infarction (abstr). $J$ Am Coll Cardiol $1991 ; 17: 152 \mathrm{~A}$

30. Magnani B, for the PAIMS Investigators. Plasminogen Activator Italian Multicenter Study (PAIMS): comparison of intravenous recombinant single-chain human tissue-type plasminogen activator (tt-PA) with intravenous streptokinase in acute myocardial infarction. J Am Coll Cardiol 1989;13:19-26.

31. Chemg WJ, Chiang CW, Kuo CT, Lee CP, Lee YS. A comparison between intravenous streptokinase and tissue plasminogen activator with early intravenous lıeparin in acute inyocardial infarction. Am Heart $J$ 1992;123:841-846.

32. White HD Norris RM, Brown MA, Brandt P, Whitlock R Wild CJ Left ven tricular end-systolic volume as the major determinant of survival after recovery from myocardial infarction. Circulation 1987;76:41-51.

33. White HD, Rivers, JT, Maslowski AH, Ormiston JA, Takayama M, Hart H, Sharpe DN, Whitlock R, Norris R. Effect of intravenous streptokinase as compared with that of tissue plasminogen activator on left ventricular function after first myocardial infarction. $N$ Engl J Med 1989;320:817-821.

34. Simoons ML, Nijssen K, de Bono D, and the European Cooperative Study Group. Thrombolytic therapy for early recurrent ischemia in myocardial infarction (abstr). Circulation 1991;84 (suppl II):II-572.

35. Smalling RW, Schumacher R, Morris D, Harder K, Fuentes F, Valentine R, Battey L, Merhige M, Pitts D, Lieberman H, Nishikawa A, Adyanthaya A, Hopkins A, Grossbard E. Improved infarct-related arterial patency after high dose, weight-adjusted, rapid infusion of tissue-type plasminogen activator in myocardial infarction: results of a multicenter randomized trial of two dosage regimens. $J$ Am Coll Cardiol 1990; 15:015-921

36. Carney RJ, Murphy GA, Brandt TR, Daley PJ, Pickering E, White HJ, McDonough TJ, Vermilya SK, Teichman SL. Randomized angiographic trial of recomhinant tissue-type plasminngen activator (alteplase) in myocardial infarction. $J \mathrm{Am}$ Coll Cardiol 1992;20:17-23.

37. Topol EJ, Morris DC, Smalling RW, Schumacher RR, Taylor CR. A multicenter, randomized, placebo-controlled trial of a new form of intravenous recom binant tissue-type plasminogen activator (Activase) in acute myocardial infarction Am Coll Cardiol 1987:9:1205-1213.

38. The TIMI Research Group. Immediate versus delayed catheterization and angioplasty following thrombolytic therapy of acute myocardial infarction. JAMA 1988:260:2849-2858.

39. Topol EJ, Ellis SG, Califf RM, George B, Stump D, Bates E, Nabel E, Walun J, Candela R, Lee KL, Kline EM, Pill B, and the TAMI 4 Study Group. Combined tissue-type plasminogen activator and prostacyclin therapy for acute myocardial infarction. J Am Coll Cardiol 1989;14:877-884.

40. Califf RM, Topol EJ, Stack RS, Ellis SG, George BS, Kereiakes D, Samaha J, Worley S, Anderson J, Woodlief L, Wall T, Phillips HR, Abbottsmith CW, Candela R, Flanagan WH, Sasahara A, Mantell S, Lee KL, for the TAMI Study Group. Evaluation of combination thrombolytic therapy and timing of cardiac catheterization in acute myocardial infarction, Results of thrombolysis and angioplasty in myocardial infarction-phase 5 randomized trial. Circulation 1991:83:1543-1556. 41. Johns JA, Gold HK, Leinbach RC, Yasuda T, Gimple LN, Werner W, Finkelstein D, Newell J, Ziskind AA, Collen D. Prevention of coronary artery reocclusion and reduction in late coronary artery stenosis after thrombolytic therapy in patients with acute myocardial infarction. Circulation 1988;78:546-556.

42. Whitlow PL, Bashore TM. Catheterization/Rescue Angioplasty Following Thrombolysis (CRAFT) study: acute myocardial infarction treated with recombinant tissue plasminogen activator versus urokinase (abstr). J Am Coll Cardiol 1991; 17(suppl):276A

43. Topol EJ, George BS, Kereiakes DJ, Stump DC, Candela RJ, Abbottsmith CW, Aronson L, Pickel A, Boswick JM, Lee KI, Ellis S, Califf RM, and the TAMI Study Group. A randomized controlled trial of intravenous tissue plasminogen acti- 
vator and early intravenous heparin in acute myocardial infarction (TAMI-3). Circulation 1989;79:281-286.

44. Grines CL, Nissen SE, Booth DC, Gurley JC, Chelliah N, Wolf R, Blankenship J, Branco MC, Bennett K, DeMaria AN, and the KAMIT Group. A prospective, randomized trial comparing combination half-dose tissue-type plasminogen activator and streptokinase with full-dose tissue-type plasminogen activator. $\mathrm{Cir}$ culation 1991;84:540-549.

45. Neuhaus KL, Tebbe U, Gotwik M, Weber M, Feurer W, Niederer W, Haerer W, Praetorius F, Grosser KD, Huhmann W, Hoepp HW, Alber G, Sheikhzadeh A, Schneider B. Intravenous recombinant tissue plasminogen activator (rt-PA) and urokinase in acute myocardial infarction: results of the German Activator Urokinase Study (GAUS). J Am Coll Cardiol 1988;12:581-587.

46. Anderson JL. Becker LC. Sorenson SG, Karagounis LA. Browne KF, Shah PK, Morris DC, Fintel DJ, Mueller HS, Ross AM, Hall SM, Askins JK, Doorey AJ, Grines CL, Moreno FL, Marder VJ. APSAC versus alteplase in acute myocardial infarction: comparative effects on left ventricular function, morbidity and 1-day coronary artery patency. J Am Coll Cardiol 1992;20:753-766.

47. The TIMI Study Group. Comparison of invasive and conservative strategies after treatment with intravenous tissue plasminogen activator in acute myocardial infarction. $N$ Engl J Med 1989;320:618-627.

49. de Bono DP, Simoons ML, Tijssen J, Arnold AE, Betriu A, Burgersdijk C, Lopez Bescos L, Mueller E, Pfisterer M, Van de Werf F. Early intravenous heparin enhances coronary patency after alteplase thrombolysis: results of a randomized double blind European Cooperative Study Group trial. Br Heart J 1992;67:122-128. 49. Thompson PL, Aylward PE, Federman J, Giles RW, Harris PJ, Hodge RL, Nelson G, Thomson A, Tonkin AM, Walsh WF, for the National Heart Foundation of Australia Coronary Thrombolysis Group. A randomized comparison of intravenous heparin with oral aspirin and dipyridamole 24 hours after recombinant tissue-type plasminogen activator for acute myocardial infarction. Circulation 1991:83:1534-1542.

80. Rapold HJ, Kuemmerdi H, Weiss M, Baur H, Haeberli A. Monitoring of fibrin generation during thrombolytic therapy of acute myocardial infarction with recombinant tissue-type plasminogen activator. Circulation 1989;79:980-989.

51. Gemmell JD, Hogg KJ, Macintyre PD, Booth N, Rae AP, Dunn FG, Hillis WS A pilot study of the efficacy and safety of bolus administration of alteplase in acute myocardial infarction. Br Heart J 1991;66:134-138.

52. Neuhaus KL, Feurer W, Jeep-Tebbe S, Niederer W, Vogt A, Tebbe U. Improved thrombolysis with a modified dose regimen of recombinant tissue-type plasminogen activator. J Am Coll Cardiol 1989;14:1566 1569.

53. Neuhaus KL, von Essen R, Tebbe U, Vogt A, Roth M, Riess M, Niederer W, Forycki F, Wirtzfeid A, Maeurer W, Limbourg P, Merx W, Haerten K. Improved thrombolysis in acute myocardial infarction with front-loaded administration of alteplase: results of the rt-PA-APSAC patency study (TAPS). $J$ Am Coll Cardiol 1992;19:885-891.

54. Passamani E, Hodges M, Herman M, Grose R, Chaitman B, Rogers W, Forman S, Terrin M, Knatterud G, Robertson T, Braunwald E, for the TIMI Investi- gators. The Thrombolysis in Myocardial Infarction (TIMI) phase II pilot study: tissue plasminogen activator followed by percutaneous transluminal coronary angioplasty. J Am Coll Cardiol 1987;10:51B-64B

55. Purvis JA, Trouton TG, Roberts M, McKeown P, Mulholland MG, Dalzel GW, Wilson C, Patterson G, Webb SW, Khan MM, Campbell NP, Adgey J. Effectiveness of double bolus alteplase in the treatment of acute myocardial infarction Am J Cardiol 1991;68:1570-1574.

56. Wall TC, Califf RM, George BS, Ellis S, Samaha J, Kereiakes D, Worley S, Sigmon K, Topol E, for the TAMI-7 Study Group. Accelerated plasminogen activator dose regimens for coronary thrombolysis. J Am Coll Cardiol 1992;19. $482-489$.

57. Kasper W, Meinertz T, Wollschlager H, Bonzel T, Wolff P, Drexler H, Hofmann T, Zeiher A, Just H. Coronary thrombolysis during acute myocardial infarction by intravenous BRL, 26921, a new anisoylated plasminogen-streptokinase activator complex. Am J Cardiol 1986:58:418-421.

58. Relik-van Wely L, Visser RF, van der Pol J, Bartholomeus I, Couvee JE, Drost H, Vet A, Klomps H, van Ekelen W, van den Berg F, Kraus XH. Angiographically assessed coronary arterial patency and reocclusion in patients with acute myocardial infarction treated with anistreplase: results of the Anistreplase Reocclusion Multicenter Study (ARMS). Am J Cardiol 1991;68:296-300.

59. Meinertz T, Kasper W, Schumacher M, Just H, for the APSAC Multicenter Trial Group. The German multicenter trial of anisoylated plasiminogen streptokinase activator complex versus heparin for acute myocardial infarction. Am J Cardiol 1988:62:347-351.

60. ISAM. A prospective trial of intravenous streptokinase in acute myocardial infarction. Mortality, morbidity and infarct size at 21 days. N Engl I Med 1986;314 $1465-1471$.

61. Ohman EM, Califf RM, Topol EJ, Candela R, Abbottsmith C, Ellis S, Sigmon KN, Kereiakes D, George B, Stack R, and the TAMI Study Group. Consequences of reocclusion after successful reperfusion therapy in acute myocardial infarction. Circulation 1990;82:781-791.

62. Hsia J, Hamilton W, Kleiman N, Roberts R, Chaitman B, Ross A, for the HART Investigators. A comparision between heparin and low dose aspirin as adjunctive therapy with tissue plasminogen activator for acute myocardial infarction. $N$ Engl $J$ Med 1990;323:1433-1437.

63. Braunwald E, Kloner R. The stunted myocardium: prolonged, postischemic ventricular dysfunction. Circulation 1982;66:1146-1149.

64. Harrison KJ, Califf RM, Woodlief L, Kereiakes D, George BS, Stack RS, Ellis S, Lee KL, O'Neill W, Topol EJ, and the TAMI Study Group. Systolic left ven tricular function after reperfusion therapy for acute myocardial infarction. Circula tion 1993;87:1531-1541.

65. Sabia PJ. Powers ER, Ragosta M, Sarembock IJ, Burwell L, Kaul S. An association between collateral blood flow and myocardial viability in patients with recent myocardial infarction. $N$ Engl J Med 1992;327:1825-1831.

68. Van de Werf F. Discrepancies between the effects of coronary reperfusion on survival and left ventricular function. Lancet 1989;1:1367-1369. 